Article

\title{
Power Transmission Scheduling for Generators in a Deregulated Environment Based on a Game-Theoretic Approach
}

\author{
Bingtuan Gao ${ }^{1,2, *}$, Tingting $\mathrm{Ma}^{1}$ and Yi Tang ${ }^{1}$ \\ Received: 10 September 2015; Accepted: 2 December 2015; Published: 5 December 2015 \\ Academic Editor: David Wood \\ 1 School of Electrical Engineering, Southeast University, Nanjing 210096, China; \\ matingting9414@163.com (T.M.); tangyi@seu.edu.cn (Y.T.) \\ 2 Jiangsu Key Laboratory of Smart Grid Technology and Equipment, Nanjing 210096, China \\ * Correspondence: gaobingtuan@seu.edu.cn; Tel. +86-25-8379-4163; Fax: +86-25-8379-0617
}

\begin{abstract}
In a deregulated environment of the power market, in order to lower their energy price and guarantee the stability of the power network, appropriate transmission lines have to be considered for electricity generators to sell their energy to the end users. This paper proposes a game-theoretic power transmission scheduling for multiple generators to lower their wheeling cost. Based on the embedded cost method, a wheeling cost model consisting of congestion cost, cost of losses and cost of transmission capacity is presented. By assuming each generator behaves in a selfish and rational way, the competition among the multiple generators is formulated as a non-cooperative game, where the players are the generators and the strategies are their daily schedules of power transmission. We will prove that there exists at least one pure-strategy Nash equilibrium of the formulated power transmission game. Moreover, a distributed algorithm will be provided to realize the optimization in terms of minimizing the wheeling cost. Finally, simulations were performed and discussed to verify the feasibility and effectiveness of the proposed non-cooperative game approach for the generators in a deregulated environment.
\end{abstract}

Keywords: power transmission scheduling; non-cooperative game; wheeling cost; congestion cost

\section{Introduction}

Over the past few years, the electric power industry in China has experienced a strong drive toward deregulation. In 2014, transmission and distribution electricity pricing reform has been carried out in Shenzhen as the document "The notice about the reform pilot of transmission and distribution price carried out in Shenzhen" issued by the National Development and Reform Commission of China [1], which would deregulate the power generation side market and the demand side market. Once both sides of the market are opening, the role of the electric power utilities in China will change from traditionally buying and selling electricity to only providing transmission service for generators and end users, which also means that the electricity pricing mechanism in China will be changed dramatically. Reasonable pricing mechanisms based on open electricity markets is essential to achieve optimal allocation of resources, lower energy cost, higher service quality, and so on. Moreover, establishing appropriate independent transmission electricity pricing mechanism is still an open problem for deregulated electricity markets all over the world [2].

Independent transmission electricity price is based on wheeling cost. Wheeling is defined as "the use of a utility's transmission facilities to transmit power for other buyers and sellers" [3]. The buyer or seller pays transmission cost for the usage of utility's transmission facilitates. This transmission cost is also called wheeling cost [4]. Wheeling cost is mainly used for recovery of investment and costs 
of operation, management and staff, in order to maintain good operation and development of the transmission system. Wheeling cost is reasonable and affects not only the survival and development of electric utilities but also the benefit of generators and end users. Various algorithms and methods have been used and proposed to evaluate the cost of transmission services. The current methods can be mainly classified into two categories [2]: embedded cost of wheeling methods [3,5-7] and marginal cost pricing method [8-10]. Embedded cost of wheeling methods, including the postage stamp method, contract path method, boundary flow methods and so on, recover the embedded capital costs and the average annual operating and maintenance costs of existing facilities from a particular wheeling transaction. The marginal cost pricing method is based on microeconomics. Additionally, Nojeng et al. [11] proposed an improved MW-Mile method by considering not only the changes in MW flows but also the quality of the load, i.e., the power factor. Monsef et al. [12] proposed a reliability-based method for allocating the cost of transmission networks. Moreover, a two-step method based on the perfect coupling of the circuit theory with the Aumann-Shapley method is proposed in [13]. The method can calculate allocation costs for each branch of the transmission system to identify and quantify the individual responsibility of generators and loads.

Although the aforementioned papers can calculate wheeling cost under several different conditions, most of them missed studying the effect of congestion cost, which should be covered by wheeling cost. Congestion is defined as the overloading of one or more transmission lines and/or transformers in the power system [14]. In order to solve the problem of congestion, particle swarm optimization (PSO) methods $[14,15]$ and bacterial foraging algorithms $[16,17]$ were successfully implemented. Kanwardeep Singh et al. [18] presented an effective methodology for congestion management in deregulated power system networks considering optimal placement of a distributed generator based on bus impedance matrix considering contribution factors. Harry Singh et al. [19] proposed a pool model and a bilateral model to deal with the transmission congestion costs in competitive market and evaluated some aspects of the models based on game theory. Erli et al. [20] proposed a cooperative game based scheme for cost allocation of transmission line expansion to tackle with congestion problem in the network.

This paper deals with a scenario in a deregulated environment of power market having multiple generators, multiple transmission lines, and one aggregated end user. The generators have to compete with each other to optimize their profit. By considering the game theory-based/double auction-based approaches and control theory-based approaches [21-24], we formulate wheeling pricing including congestion cost for the players in the deregulated environment of power market. To make their electricity more competitive in the end users market, a game-theoretic approach is proposed for generators to schedule their power transmission effectively to lower the wheeling costs. The main contributions of this paper can be summarized as: (1) by assuming generators are selfish and rational, a non-cooperative game approach is proposed for multiple generators to lower their wheeling costs, where strategies of the non-cooperative game are the power transmission scheduling of the generators; (2) a distributed algorithm is presented to realize the optimization in terms of minimizing the wheeling cost, which can be guaranteed at the Nash equilibriums of the formulated non-cooperative games; (3) simulations are performed to verify the effectiveness of the proposed approach, and discussions show that all the generators, electric utilities, and aggregated end users can benefit from the game.

The rest of the paper is organized as follows. Section 2 presents the method that this paper adopts for the pricing model of the wheeling cost. In Section 3, the novel proposed method of this paper for optimizing wheeling cost based on the game-theoretic method is presented. Simulation results are presented and discussed in Section 4. Finally, conclusions are provided in Section 5. 


\section{System Model}

\subsection{Wheeling Cost Model}

As shown in Figure 1, we consider a typical power system consisting of a set of $\mathcal{N}=\{1, \ldots, N\}$ generators, a set of $\mathcal{M}=\{1, \ldots, M\}$ transmission lines managed by different wheeling utilities and different end users. Each generator $n \in \mathcal{N}$ can supply electrical power to the end users in a certain area via a single transmission line or several transmission lines. Generator $n \in \mathcal{N}$ can be a classical thermal power plant, a renewable solar power station or a wind farm, and other new energy power plant. Transmission lines belongs to utility companies who provide the power transmission service. End users are taken as a whole in this paper. Generators and/or users have to pay the wheeling cost to electric utilities.

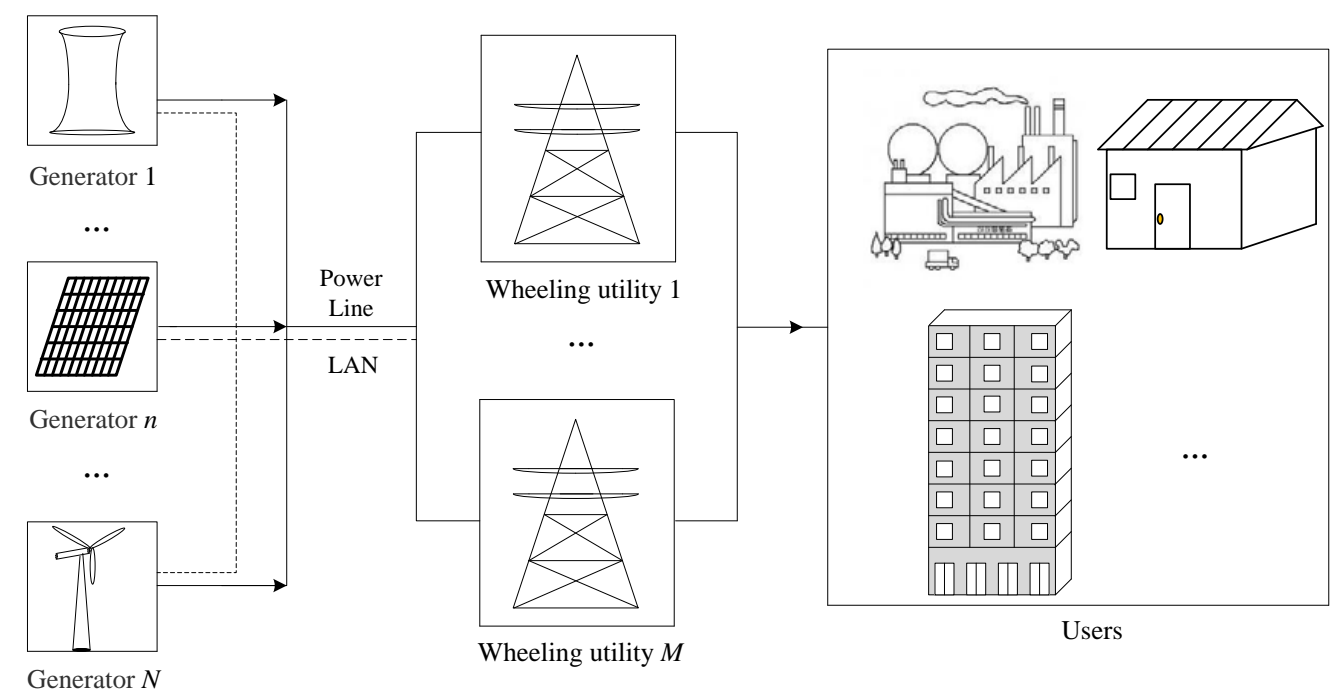

Figure 1. Figure of wheeling transaction.

Based on the embedded cost method [11,25], wheeling cost can be calculated as

$$
G(q)=R+W(q)+Z(q)
$$

where $q$ denotes transmitted electricity energy, $R$ is the cost of transmission capacity which reflects investment and maintenance cost of electric utilities, $W(q)$ is the cost of losses, $Z(q)$ is the congestion cost.

Electric utilities make profits only from wheeling cost paid by generators. Accounting for a large proportion of wheeling cost, cost of transmission capacity directly relates to whether the investment of electric utilities can be recycled. Cost of transmission capacity generators need to pay can be calculated as:

$$
R=\frac{1}{M} \sum_{m=1}^{M}\left(\frac{T_{m} \cdot \frac{r}{(1+r)^{t}-1}}{Q_{m, \text { year }}}\right)
$$

where $T_{m}$ is investment cost of transmission line $m \in \mathcal{M}, r$ is discount rate, $t$ is recovery period, $Q_{m, y e a r}$ is the predicted transmission capacity of transmission line $m$ during one year. For the line loss in Equation (1), it can be calculated as:

$$
W(q)=C(q) \times \sigma
$$

where $C(q)$ is the generation cost, and $\sigma$ is line loss rate, which is widely used by electric utilities to solve the allocation problem of loss cost. 
If user loads are stable, load curve can be modified into a polygonal line. Each segment denotes a period of time, and load is invariable during this time. Inspired by [25], congestion cost can be described as:

$$
Z(q)=R\left(\ln \frac{\alpha(q)}{\beta(q)}\right) e^{\beta(q)}
$$

where

$$
\alpha(q)=\frac{q_{m, a l l}}{q_{m, \max }}, \beta(q)=\frac{q_{n, m}}{q_{m, \max }}
$$

and $q_{m, \max }$ is maximum transmission capacity of transmission line $m, q_{m, \text { all }}$ is power flow on transmission line $m$ of all generators, $q_{n, m}$ is power flow related to generator $n$ on transmission line $m$. Therefore, one can see $q_{m, \text { all }}=\sum_{n=1}^{N} q_{n, m}$ straightforwardly. Logarithmic term and exponential term of Equation (4) denote two mathematical models: the former is congestion extent in branch caused by generator $n$, and the latter is the proportion of power flow caused by generator $n$ in power flow on transmission line $m$. During the period under heavy load, congestion cost will be high. The additional cost caused by congestion can be regarded as the fund used to improve the transmission capacity of transmission lines. Accordingly, congestion cost will be low and even be negative during the period under light load. In other words, congestion cost can be effectively reduced by alleviating congestion. Under this condition, more generators can be encouraged to use transmission lines with light-loads to transmit power. It should be noted that wheeling cost cannot be negative practically even though there is no congestion on transmission line. Therefore, constraint $Z(q) \geq 0$ should be satisfied.

\subsection{Cost Model of Generators}

Assuming that the daily power consumption of users is predicted by electric utilities, and total energy generated by all generators are determined by electric utilities. Thus, balance of supply and demand in power system can be guaranteed. If the generated electric energy production is only determined by generators or regulated by the market, an imbalance between supply and demand may occur. However, the imbalance is harmful for stability of power system. Generation cost of generators can be divided into two parts: the fixed cost and the variable cost. For example, considering thermal power plants, the fixed cost mainly consists of capacity cost, which does not change with different generations. Variable cost of thermal power plant mainly consists of fuel consumption, which changes with different generations.

For each generator $n$, let $q_{n}^{h}$ denote the generated energy at time slot $h \in \mathcal{H}=\{1, \cdots, H\}$. For a daily operation of the grid, each time slot may take $15 \mathrm{~min}$ and we have $H=96$. Generation scheduling for generator $n$ in a whole day is denoted by $\mathbf{q}_{n} \triangleq\left[q_{n}^{1}, \cdots, q_{n}^{h}, \cdots, q_{n}^{H}\right]$. Let $C_{n}\left(\mathbf{q}_{n}\right)$ denote the cost of generating electricity by each generator $n \in \mathcal{N}$, which can be modeled as the following quadratic function [26]:

$$
C_{n}\left(\mathbf{q}_{n}\right)=a_{n} \mathbf{q}_{n}^{2}+b_{n} \mathbf{q}_{n}+c_{n}
$$

where $a_{n}>0, b_{n}>0, c_{n}>0$ are fixed parameters; $c_{n}$ denotes fixed cost; $a_{n} \mathbf{q}_{n}^{2}+b_{n} \mathbf{q}_{n}$ denotes variable cost.

Although generators cannot decide the electric energy production, we assume that generators can determine the quantity of electricity transported on each transmission line. For each transmission line $m \in \mathcal{M}=\{1, \ldots, M\}$, we define a power transmission scheduling vector as:

$$
\mathbf{x}_{n, m}=\left[x_{n, m}^{1}, \cdots x_{n, m}^{h}, \cdots x_{n, m}^{H}\right]
$$

where $x_{n, m}^{h}$ denotes the proportionality coefficient decided by generator $n, x_{n, m}^{h} \in[0,1]$. The proportionality coefficient is the proportion of energy transported by transmission line $m$ in total 
energy generated by generator $n$ at time slot $h$. Note that total electricity will be transmitted to users by $M$ transmission lines. Thus, for each generator $n$, the following constraint has to be satisfied:

$$
\sum_{m=1}^{M} x_{n, m}^{h}=1, \forall h \in \mathcal{H}
$$

The proportion of energy transported by transmission line $m$ for each generator $n$ needs to be constrained. Let $\lambda_{n, m}^{\min }$ denote the minimum proportion and $\lambda_{n, m}^{\max }$ denote the maximum proportion.

$$
\lambda_{n, m}^{\min } \leq x_{n, m}^{h} \leq \lambda_{n, m}^{\max }, \forall h \in \mathcal{H}
$$

In order to guarantee stability of power system, we suppose energy generation equal to energy consumption at each time slot $h \in \mathcal{H}$, namely,

$$
\sum_{n=1}^{N} q_{n}^{h}=E^{h}
$$

where $E^{h}$ denotes total energy consumption of end users at time slot $h$. By introducing vector $\mathbf{x}_{n}$ as power transmission scheduling on all transmission lines $m \in \mathcal{M}$, a power transmission scheduling set can be described as:

$$
\mathcal{X}_{n}=\left\{\mathbf{x}_{n} \mid \sum_{n=1}^{N} q_{n}^{h}=E^{h}, \lambda_{n, m}^{\min } \leq x_{n, m}^{h} \leq \lambda_{n, m}^{\max }, \forall h \in \mathcal{H}\right\}
$$

Let $\mathbf{q}_{n, m}$ be electric quantity transported by transmission line $m$ of each generator $n \in \mathcal{N}$.

$$
\mathbf{q}_{n, m}=\mathbf{x}_{n, m} \mathbf{q}_{n}^{\mathrm{T}}
$$

Consequently, the total power transmission of transmission line $m$ is calculated as:

$$
\mathbf{q}_{m, a l l}=\sum_{n=1}^{N} \mathbf{x}_{n, m} \mathbf{q}_{n}^{\mathrm{T}}
$$

We define a vector $\mathbf{c}_{n, m}$ is electric quantity transported by transmission line $m$ of all generators except generator $n$.

$$
\mathbf{c}_{n, m}=\sum_{i=1, i \neq n}^{N} \mathbf{x}_{i, m} \mathbf{q}_{n}^{\mathrm{T}}
$$

In other words, cost of losses $W$ and congestion cost $Z$ in Equation (1) can be regarded as functions of $\mathbf{x}_{n, m} \mathbf{q}_{n}^{\mathrm{T}}$. By assuming $\mathbf{q}_{n}$ is constant, congestion cost in Equation (4) for generator $n$ on transmission line $m$ can be written as:

$$
Z\left(\mathbf{x}_{n, m}\right)=R\left(\ln \frac{\mathbf{x}_{n, m} \mathbf{q}_{n}^{\mathrm{T}}+\mathbf{c}_{n, m}}{\mathbf{x}_{n, m} \mathbf{q}_{n}^{\mathrm{T}}} e^{\frac{\mathbf{x}_{n, m} \mathbf{q}_{n}^{\mathrm{T}}}{\bar{q}_{m, m a x}}}\right)
$$

Meanwhile, cost of line loss in Equation (3) for generator $n$ on transmission line $m$ can be written as:

$$
W\left(\mathbf{x}_{n, m}\right)=C_{n}\left(\mathbf{x}_{n, m} \mathbf{q}_{n}^{\mathrm{T}}\right) \times \sigma
$$

Because the denominator cannot be $0, \mathbf{x}_{n, m} \neq 0, \mathbf{q}_{n, m} \neq 0$. Note that $\mathbf{x}_{n, m} \geq 0, \mathbf{q}_{n, m} \geq 0$, where generated energy cannot be negative, namely, $\mathbf{x}_{n, m}>0, \mathbf{q}_{n, m}>0$. The constraint $\mathbf{x}_{n, m} \neq 0$ will not obviously influence the validity of our study, because the optimal choices for generators generally 
transport power through all transmission lines. Then, let $G\left(\mathbf{x}_{n, m}\right)$ denote wheeling cost of generator $n$ on transmission line $m$, which can be calculated as:

$$
G\left(\mathbf{x}_{n, m}\right)=R+W\left(\mathbf{x}_{n, m}\right)+Z\left(\mathbf{x}_{n, m}\right)
$$

Therefore, total cost of each generator $n \in \mathcal{N}$ is

$$
P_{n}=C_{n}\left(\mathbf{q}_{n}\right)+\sum_{m=1}^{M} G\left(\mathbf{x}_{n, m}\right)
$$

When cost of generator is reduced, the electric price can be reduced for users. It can also make generators be more competitive.

\section{Non-cooperative Game-Theoretic Approach}

In this section, based on the wheeling cost in Equation (18) for each generator, a non-cooperative game among generators is proposed to reduce wheeling cost by optimizing power transmission scheduling. And also a distributed optimization algorithm is provided to realize the optimization in terms of minimizing the wheeling cost.

\subsection{Non-Cooperative Game}

Our goal is to find the optimal scheduling of generators that minimize the wheeling cost. The optimization problem can be expressed as follows:

$$
\underset{\mathbf{x}_{n} \in \mathcal{X}_{n}, \forall n \in \mathcal{N}}{\operatorname{minimize}} \sum_{h=1}^{H} P_{n}\left(\sum_{m \in M} x_{n, m}^{h}\right)
$$

The optimization problems defined in Equation (19) can be solved to obtain an optimal solution for the given generators and their power transmission scheduling. Each generator selects strategy to make probability that congestion occurs on transmission lines as low as possible. Accordingly, wheeling cost of the generator will decrease and congestion can also be effectively alleviated and even avoided.

Generally, each generator behaves in a selfish and rational way, and attempts to maximize the steady-state value of payoff [27]. Therefore, the interaction among the generators can be modeled as a non-cooperative game, whose solution is the well-known Nash equilibrium. We formulate a non-cooperative game among generators, $\Xi=\left\{\mathcal{N},\left\{\mathcal{X}_{n}\right\}_{n \in \mathcal{N}},\left\{S_{n}\right\}_{n \in \mathcal{N}}\right\}$, which is characterized by three main elements as following:

- Players: Generators in the set $\mathcal{N}$.

- Strategies: Each generator $n \in \mathcal{N}$ selects its power transmission strategy to maximize its payoff.

- Payoffs: Gains and costs from adjusting power transmission scheduling for each generator $n \in \mathcal{N}$. Given a certain strategy choice $\mathbf{x}_{n}$ by any generator, the utility function can be characterized by:

$$
S_{n}\left(\mathbf{x}_{n}, \mathbf{x}_{-n}\right)=-P_{n}=-C_{n}\left(\mathbf{q}_{n}\right)-\sum_{m=1}^{M} G\left(\mathbf{x}_{n, m}\right)
$$

where $\mathbf{x}_{-n} \triangleq\left[\mathbf{x}_{n}, \ldots, \mathbf{x}_{n-1}, \mathbf{x}_{n+1}, \ldots, \mathbf{x}_{n}\right]$ denotes the power transmission scheduling for all generators except the generator $n$.

Based on the definitions of the strategy and payoff in the game, all generators will try to adjust their power transmission scheduling to minimizing their wheeling costs until a Nash equilibrium of the game is reached: 
- Nash equilibrium: Consider the game played among a set $\mathcal{N}$ of players. The power transmission scheduling $\mathbf{x}_{n} \in \mathcal{X}_{n}$ for each generator $n \in \mathcal{N}$ form a unique Nash equilibrium if and only if we have:

$$
S_{n}\left(\mathbf{x}_{n}^{*}, \mathbf{x}_{-n}^{*}\right) \geq S_{n}\left(\mathbf{x}_{n}, \mathbf{x}_{-n}^{*}\right)
$$

It means that once the power transmission game is at its unique Nash equilibrium, no one will benefit by deviating from $\mathbf{x}_{n}^{*}, \forall n \in \mathcal{N}$. Although the existence of Nash equilibrium is not guaranteed for any non-cooperative game in general, we can obtain the following existence result for the proposed game [28].

Theorem 1: For the non-cooperative game $\Xi=\left\{\mathcal{N},\left\{\mathcal{X}_{n}\right\}_{n \in \mathcal{N}},\left\{S_{n}\right\}_{n \in \mathcal{N}}\right\}$, there exists a unique pure-strategy Nash equilibrium.

Theorem 2: The unique Nash equilibrium of game $\Xi$ is the optimal solution of problem in Equation (19). The proof of Theorem 1 and Theorem 2 are given in Appendix A and Appendix B, respectively.

\subsection{Algorithm for the Wheeling Cost Game}

According to the proof in the Appendix, the problem in Equation (19) is concave and admits an unique optimal solution. The algorithm basically allows each player to play his best response strategy, announce only his transmission scheduling, and repeat until equilibrium is reached. The algorithm is explained in detail in Algorithm 1. First, it start with some random power transmission schedules. Each step will result in the total wheeling cost decreasing or remaining the same. Since the objective function is bounded from below, the iterations will eventually converge to a fixed point which wheeling cost $P_{n}$ up to some accuracy $\varepsilon$. Once generators reach this point, they will have no preference to change since the NE is unique, and convergence is achieved.

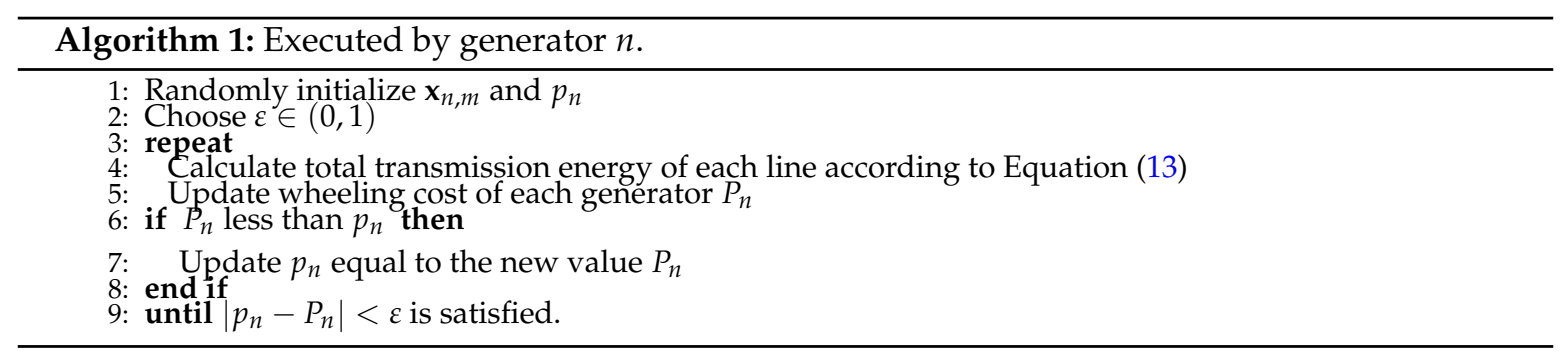

\section{Simulation and Discussion}

\subsection{Simulation Results}

This section presents several simulations of the method presented in the previous section on the problem of wheeling cost optimization. We assume $N=8$ in Figure 1, and eight generators are two wind farms, five solar power stations and one thermal power plants, respectively. For simplification of simulation, two wind farms are taken as a whole, meanwhile, five solar power stations are taken as a whole. Installed capacity of thermal power plant is $5000 \mathrm{MW}$, and total installed capacity of two wind farms and five solar power stations are both $1500 \mathrm{MW}$. The eight generators supply power to users through two transmission lines, supposing that line 1 with 9000 MW of power transmission capacity and line 2 with $5000 \mathrm{MW}$ of power transmission capacity. In order to promote generation of electricity by new energy, all electricity produced by wind farms and solar power stations will be transported to the users preferentially.

For simplicity, it is assumed that generation cost function of thermal power plant is $C_{1}\left(q_{1}\right)=0.018 q_{1}^{2}+1.7 q_{1}$, generation cost function of wind farm is $C_{2}\left(q_{2}\right)=0.022 q_{2}^{2}+1.7 q_{2}$, generation cost function of solar power station is $C_{3}\left(q_{3}\right)=0.021 q_{3}^{2}+2.1 q_{3}$. Daily load curve of users is shown in Figure 2. One day is divided into 96 time slots, electricity price updates every $15 \mathrm{~min}$. Suppose that the sum of electricity generated by three power plants is consistent with the daily load 
curve of users every time, which also means that supply and demand obtain a balance. Electricity generated by solar power stations and wind farms are shown in Figure 3. Then electricity generated by thermal power plant is the difference between loads of users and total electricity generated by wind farms and solar power stations. Parameters of wheeling cost model are as follows: investment cost of transmission line $1 T_{1}=2 \times 10^{6}$, investment cost of transmission line $2 T_{2}=1.8 \times 10^{6}$, discount rate $r=12 \%$, line loss rate $\sigma=5 \%$, accuracy $\varepsilon=1$. Before optimization, electricity of generated by each plant will be divided equally to transmit through the two transmission lines at every moment, hence, $x_{n, m}^{h}=0.5$ for each generator.

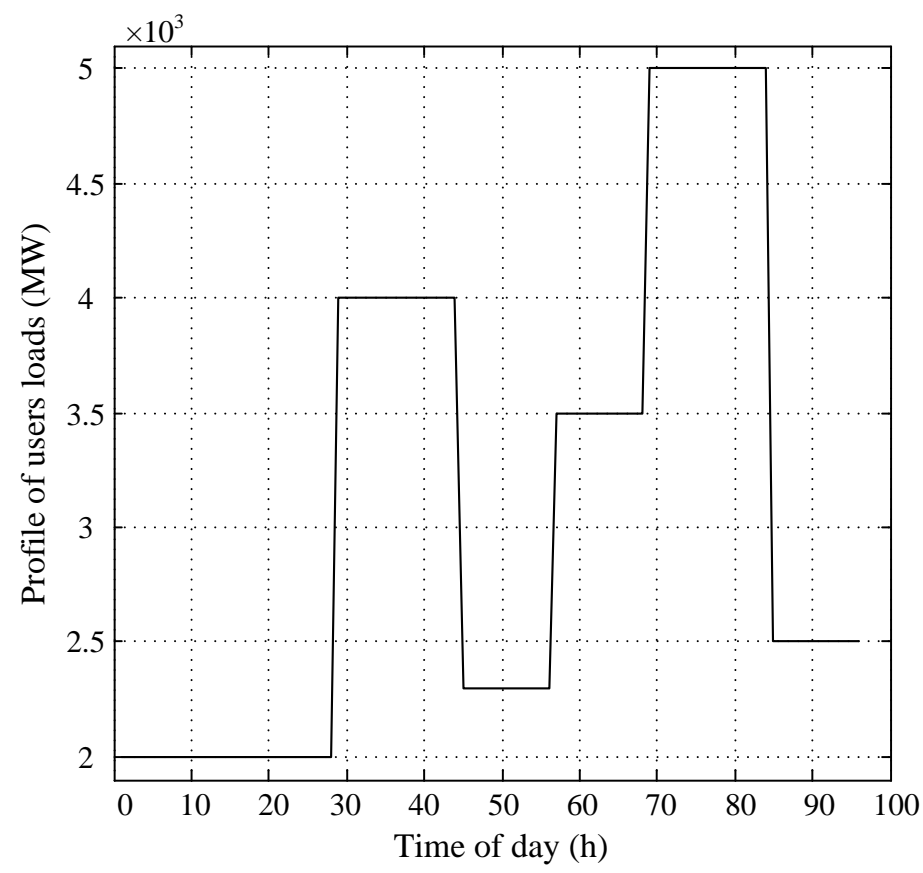

Figure 2. Daily load curve of users.

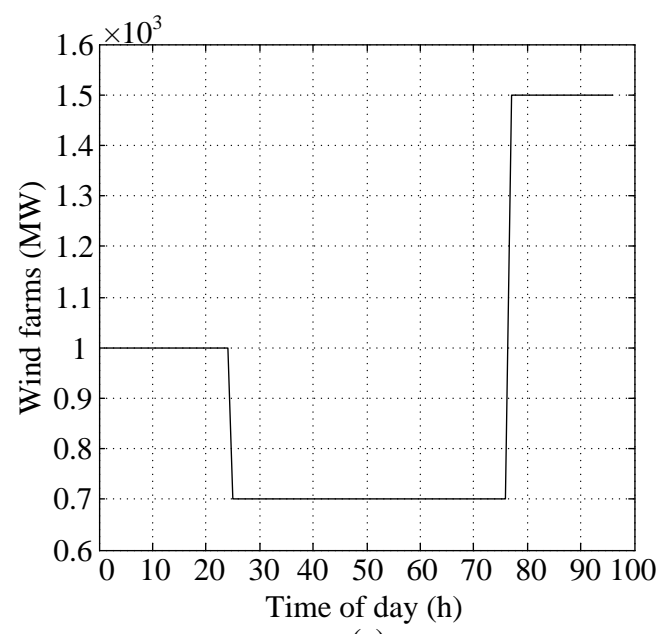

(a)

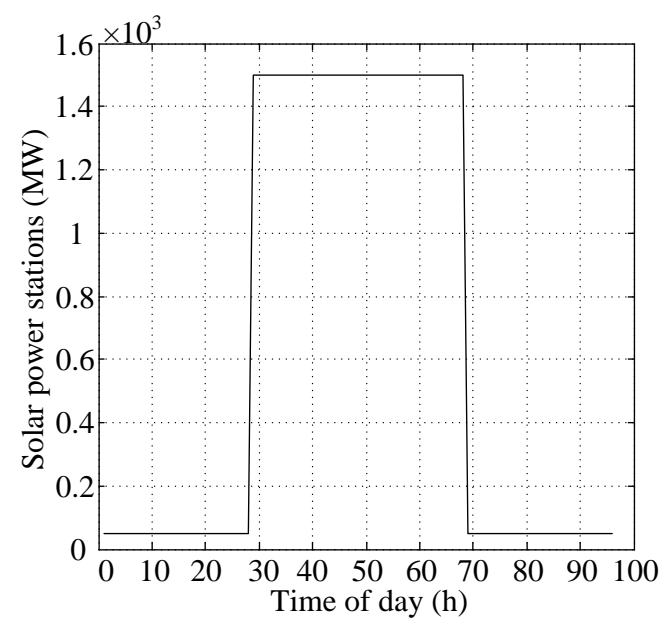

(b)

Figure 3. Generation of electricity: (a) Wind farms; (b) Solar power stations.

By using the proposed method, the non-cooperative game among generators can be implemented automatically with the distributed algorithm. Figure 4 shows the quantity of electricity transported through each transmission line after optimization, which generated by wind farms, solar 
power stations and thermal power plant, respectively. From the figure, one can see that quantity of electricity transported through transmission line 1 is large because of large transmission capacity. Table 1 shows daily wheeling cost of three power plants, including cost before and after optimization, where $\Delta P_{n}$ means the difference of wheeling cost between before and after optimization for each typical generator. One can see that wheeling costs of generators are all decreased, which validates the effectiveness and efficiency of the proposed optimal algorithm in this paper. Therefore, all the generators will be benefited and willing to take part in the game. Figure $5 \mathrm{a}-\mathrm{c}$ denotes wheeling cost of wind farms, solar power stations and thermal power plant with and without game, respectively. Wheeling cost with game is lower than wheeling cost without game at each time, which can also validate effectiveness of algorithm presented previously. According to Figures 3 and 5, note that the wheeling cost increases obviously when quantity of transmission electricity is large. Meanwhile, the wheeling cost is low when quantity of transmission electricity is small, which can promote power transmission. It is important to highlight that when congestion is alleviated effectively, stability of power system is guaranteed. Besides, service life of transmission lines will be extended, which is beneficial for electric utilities because of the reduced fixed cost.

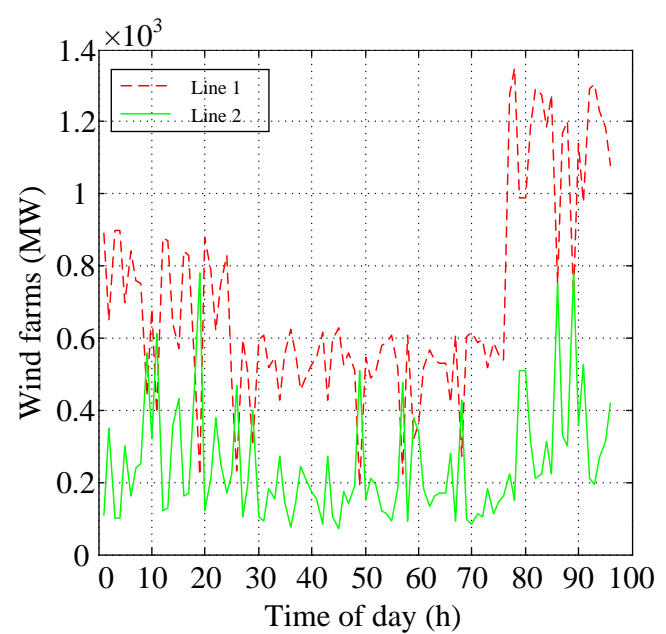

(a)

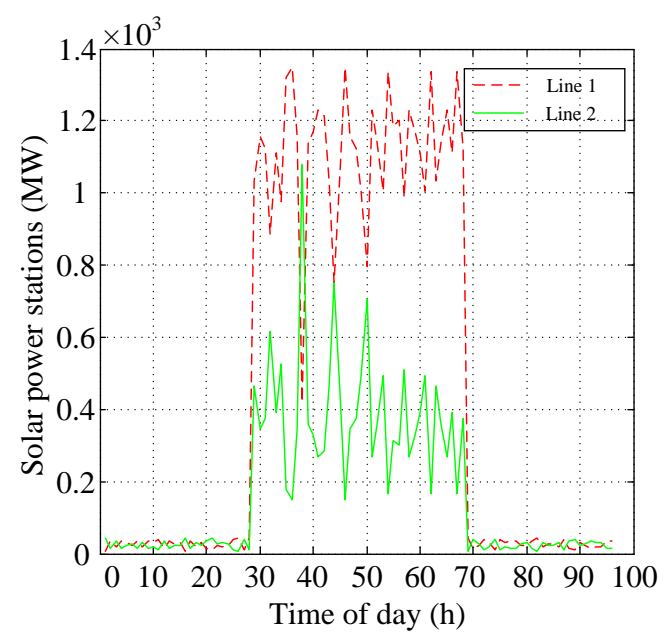

(b)

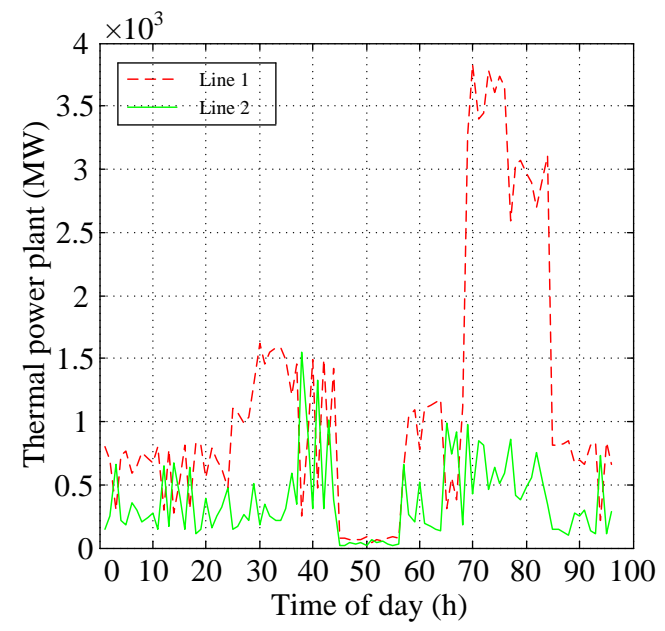

(c)

Figure 4. Electricity transported through each transmission line: (a) Wind farms, (b) Solar power stations and (c) Thermal power plant. 


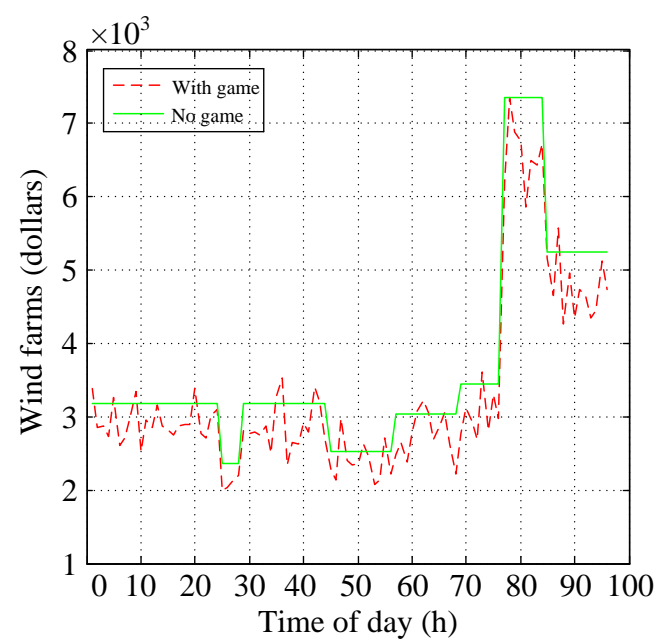

(a)

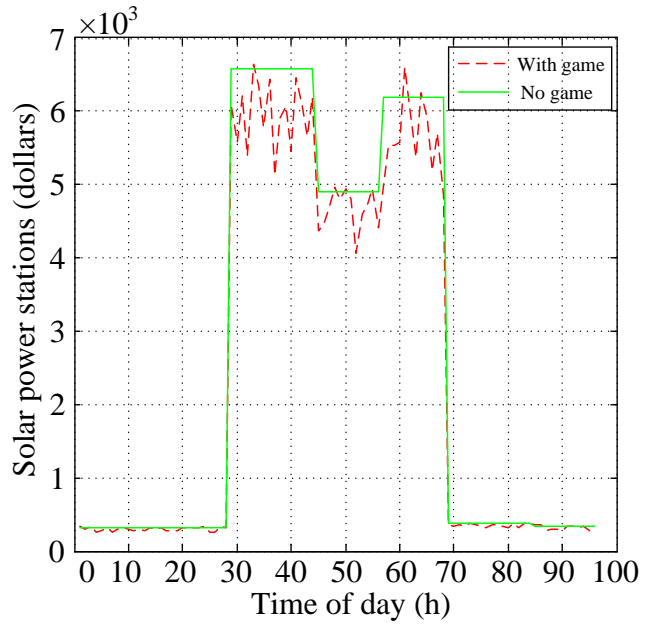

(b)

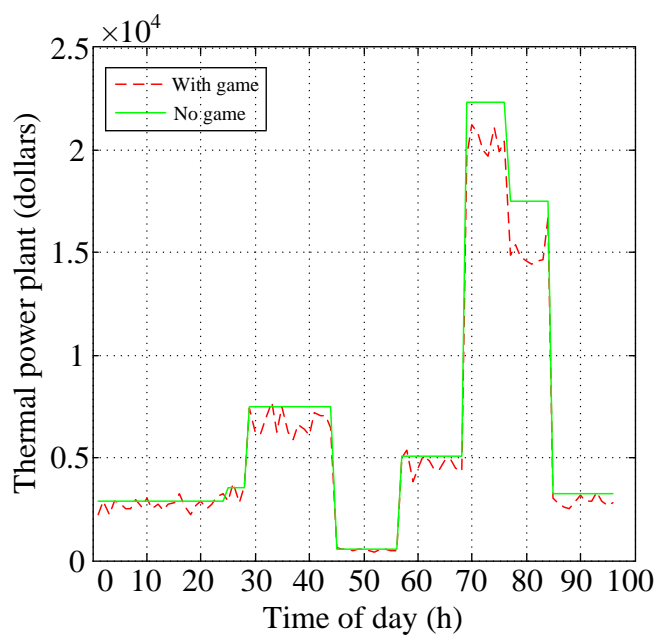

(c)

Figure 5. Wheeling cost of generators: (a) Wind farms, (b) Solar power stations and (c) Thermal power plant.

Table 1. Daily wheeling cost of each generator (dollars).

\begin{tabular}{cccc}
\hline Generators & With Game & No Game & $\boldsymbol{\Delta P}_{\boldsymbol{n}}$ \\
\hline Wind farm & 79,372 & 88,198 & 8826 \\
Solar power station & 64,681 & 68,571 & 3890 \\
Thermal power plant & 139,950 & 152,590 & 12,640 \\
\hline
\end{tabular}

The game-based method proposed previously is one decentralized control approach, in contrast with central control approach. In central control system, not only generated electric energy production of generators but power transmission scheduling is determined by utility companies. System parameters are the same as the previous case, and the total wheeling cost of generators during one day with central control is shown in Table 2. $\triangle P 1$ denotes the difference of wheeling cost between scheduling with game and naive scheduling. Similarly, $\triangle P 2$ denotes the difference of wheeling cost between scheduling with central control and naive scheduling. One can see that the wheeling cost with central control is slightly higher than the wheeling cost with decentralized control. Furthermore, the decentralized control approach based on non-cooperative games can be implemented more easily, 
since the system with central control needs a center price setter. In decentralized control systems with game, however, each generator is motivated to adjust scheduling to lower wheeling costs.

Table 2. Daily wheeling cost under different condition (dollars).

\begin{tabular}{cccccc}
\hline Condition & No Game & With Game & Central Control & $\boldsymbol{\Delta P 1}$ & $\boldsymbol{\Delta P 2}$ \\
\hline Wheeling cost & 309,359 & 284,003 & 285,675 & 25,356 & 23,684 \\
\hline
\end{tabular}

\subsection{Discussions}

Previous simulation results show that wheeling cost and total cost of each generators, $P_{n}$ will be decreased once the generators participated the game. In other words, each generator will save some costs $\Delta P$ according to the game, as shown in Table 1 . However, do the end users have any chances to benefit from the game? Normally, different generators will have its own basic profit rate, which can be assumed as $\lambda_{n}^{\text {profit }}$ for each generator. Based on the total cost of each generators $P_{n}$, the electricity payment from the end users to each generator $P_{n}^{\text {eu }}$ can be written as:

$$
P_{n}^{\text {eu }}=\left(1+\lambda_{n}^{\text {profit }}\right) P_{n}
$$

In this case, the end users can save electricity payments as the amount of $\left(1+\lambda_{n}^{\text {profit }}\right) \Delta P_{n}$ for each generator, which means the end users will save more electricity payment than the cost saved by the generator once the generator does have profit. The total saved payment of the end users because the generators that participate in the game will be $\sum_{n=1}^{N}\left(1+\lambda_{n}^{\text {profit }}\right) \Delta P_{n}$. As a result, the end users will be benefited also according to the game.

One has to notice that the profit rate of each generator $\lambda_{n}^{\text {profit }}$ would be varied in deregulated environment of electricity market. However, regardless of the variance of the profit rate, the decrease of the total cost could lead to lower electricity price for the generator, which will result in more competitive in the market in practice.

\section{Conclusions}

In this paper, we have proposed a non-cooperative game based optimizing power transmission scheduling for generators in a deregulated environment. The wheeling cost including congestion cost has been selected as the key optimizing item. Detailed modeling, existence of the Nash equilibrium, and algorithm to realize the optimization were presented. Simulations were performed to show the effectiveness and feasibility of the proposed scheme and algorithm. Simulation results and discussions show that all the generators, electric utilities, and aggregated end users can benefit from the game: (1) for the generators participated in the game, the wheeling cost and total cost will be decreased and, therefore, they will be more competitive in the market; (2) for the electric utilities, the congestion can be alleviated effectively according to the game and the stability of the power system can be improved; (3) for the end users, the electricity payments will be saved according to the decreased total cost of the generators participated in the game.

Acknowledgments: The work is financially supported by the National Science Foundation of China (51577030), the Excellent Young Teachers Programm of Southeast University (2242015R30024), and Six Talent Peaks Project of Jiangsu Province (2014-ZBZZ-001).

Author Contributions: Bingtuan Gao contributed in developing the ideas of this research, Bingtuan Gao and Tingting Ma performed this research. All of the authors were involved in preparing this manuscript.

Conflicts of Interest: The authors declare no conflict of interest. 


\section{Nomenclature}

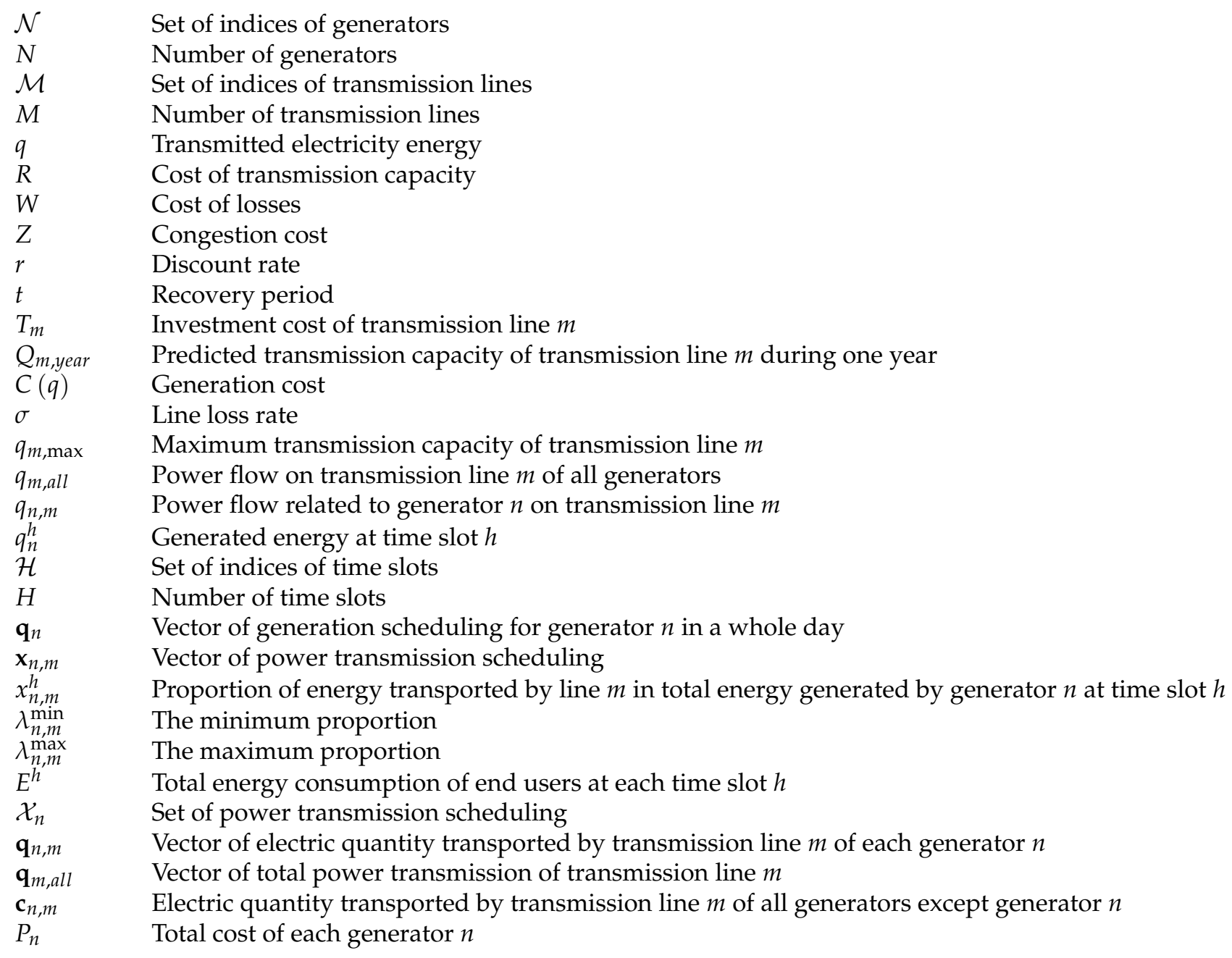

\section{Appendix A}

Assume $\mathcal{X}_{n} \subseteq R^{a}(n \in \mathcal{N})$ is a non-empty, convex and compact set. As shown in [29], if $\forall n$, $S_{n}: \mathcal{X} \rightarrow R^{1}$ is 1) graph-continuous, 2) upper semi-continuous in $x$, and 3) concave in $\mathbf{x}_{n}$, then the game $\Xi=\left\{\mathcal{N},\left\{\mathcal{X}_{n}\right\}_{n \in \mathcal{N}},\left\{S_{n}\right\}_{n \in \mathcal{N}}\right\}$ possesses a unique pure-strategy Nash equilibrium.

Note that $C_{n}\left(\mathbf{q}_{n}\right)$ is a quadratic function, it is strictly concave. Meantime, $R+W\left(\mathbf{x}_{n, m}\right)$ also is strictly concave. Therefore, if $Z\left(\mathbf{x}_{n, m}\right)$ is concave, it can be guaranteed that function $S_{n}$ is concave. In order to simplify the calculation, we use $x_{n, m}$ in place of the vector $\mathbf{x}_{n, m}$, since $x_{n, m}$ and the vector $\mathbf{x}_{n, m}$ is same for process of proof. Similarly, $c_{n, m}$ is substituted for $\mathbf{x}_{n, m}$, and $q_{n}$ is substituted for $\mathbf{q}_{n}$. Let

$$
f\left(x_{n, m}\right)=\ln \frac{x_{n, m} q_{n}+c_{n, m}}{x_{n, m} q_{n}} e^{\frac{x_{n, m} q_{n}}{q_{m, m a x}}}
$$

and

$$
\kappa_{n, m}=\frac{q_{n}}{q_{m, \max }}
$$


Therefore, we just need to prove $f\left(x_{n, m}\right)$ is concave. The partial derivative of $f$ on $x_{n, m}$ is:

$$
\frac{\partial f}{\partial x_{n, m}}=e^{x_{n, m} \kappa_{n, m}}\left[\frac{-c_{n, m}}{x_{n, m}\left(x_{n, m} q_{n}+c_{n, m}\right)}+\kappa_{n, m} \ln \frac{x_{n, m} q_{n}+c_{n, m}}{x_{n, m} q_{n}}\right]
$$

Then, we define:

$$
g\left(x_{n, m}\right)=\frac{-c_{n, m}}{x_{n, m}\left(x_{n, m} q_{n}+c_{n, m}\right)}+\kappa_{n, m} \ln \frac{x_{n, m} q_{n}+c_{n, m}}{x_{n, m} q_{n}}
$$

thus,

$$
\begin{gathered}
\frac{\partial f}{\partial x_{n, m}}=e^{x_{n, m} \kappa_{n, m}} g\left(x_{n, m}\right) \\
\frac{\partial g}{\partial x_{n, m}}=\frac{c_{n, m}\left(2 x_{n, m} q_{n}+c_{n, m}\right)}{x_{n, m}^{2}\left(x_{n, m} q_{n}+c_{n, m}\right)^{2}}-\frac{c_{n, m} \kappa_{n, m}}{x_{n, m}\left(x_{n, m} q_{n}+c_{n, m}\right)} \\
=\frac{c_{n, m}\left[2 x_{n, m} q_{n}+c_{n, m}-\kappa_{n, m} x_{n, m}\left(x_{n, m} q_{n}+c_{n, m}\right)\right]}{x_{n, m}^{2}\left(x_{n, m} q_{n}+c_{n, m}\right)^{2}}
\end{gathered}
$$

Since the power transported by transmission line cannot be larger than the transmission capacity of the line, $\kappa_{n, m} x_{n, m}<1$. In addition, due to the fact that $x_{n, m}>0, q_{n}>0$,

$$
2 x_{n, m} q_{n}+c_{n, m}-\kappa_{n, m} x_{n, m}\left(x_{n, m} q_{n}+c_{n, m}\right)>x_{n, m} q_{n}+c_{n, m}-\kappa_{n, m} x_{n, m}\left(x_{n, m} q_{n}+c_{n, m}\right)>0
$$

Namely,

$$
\frac{\partial g}{\partial x_{n, m}}>0
$$

We can get:

$$
\frac{\partial^{2} f}{\partial x_{n, m}^{2}}=\kappa_{n, m} e^{x_{n, m} \kappa_{n, m}} g\left(x_{n, m}\right)+e^{x_{n, m} \kappa_{n, m}} \frac{\partial g}{\partial x_{n, m}}
$$

It means that, $f\left(x_{n, m}\right)$ is concave when

$$
\kappa_{n, m} g\left(x_{n, m}\right)+\frac{\partial g}{\partial x_{n, m}}>0
$$

Hence, Equation (A10) is another constraint of the non-cooperative game, and the game $\Xi=\left\{\mathcal{N},\left\{\mathcal{X}_{n}\right\}_{n \in \mathcal{N}},\left\{S_{n}\right\}_{n \in \mathcal{N}}\right\}$ possesses a unique pure-strategy Nash equilibrium when it satisfies all required conditions. In other words, the non-cooperative game among generators can obtain an equilibrium when all constraints proposed in our paper are satisfied.

\section{Appendix B}

For notational simplicity, let $\mathbf{x}_{1}^{*}, \cdots, \mathbf{x}_{N}^{*}$ denote the optimal solution of the problem in Equation (19). Besides, define:

$$
P^{*}=\sum_{h=1}^{H} P\left(\sum_{m \in \mathcal{M}} \sum_{n \in \mathcal{N}} x_{n, m}^{h *}\right)
$$

By the definition of optimality, for each generator $n \in \mathcal{N}$, one can see that

$$
P^{*} \leq \sum_{h=1}^{H} P\left(\sum_{m \in \mathcal{M}} \sum_{i \in \mathcal{N} \backslash\{n\}} x_{i, m}^{h *}+\sum_{m \in \mathcal{M}} x_{n, m}^{h}\right)
$$

together with the payoff definition of the game in Equation (20), multiplying both sides in Equation (A12) by -1 , one can get the necessary and sufficient condition of Nash equilibrium in 
Equation (21) directly. Note that game $\Xi$ has a unique Nash equilibrium. Therefore, the Nash equilibrium of game $\Xi$ is the optimal solution of problem in Equation (19).

\section{References}

1. Electricity price reform project group of economic and technical research institute of Hubei electric power company. Enlightenment of reform pilot of transmission and distribution price in Shenzhen. China Power Enterp. Manag. 2015, 13, 58-63. (in Chinese)

2. Lee, W.J.; Lin, C.H.; Swift, L.D. Wheeling charge under a deregulated environment. IEEE Trans. Ind. Appl. 2001, 37, 178-183.

3. Happ, H.H. Cost of wheeling methodologies. IEEE Trans. Power Syst. 1994, 9, 147-156.

4. Bashian, A.; Hojat, M.; Javidi, M.H.; Golmohamadi, H. Security-based tariff for wheeling contracts considering fair congestion cost allocation. J. Control Autom. Electr. Syst. 2014, 25, 368-380.

5. Kumar, N.; Reddy, Y.R.V.; Das, D.; Padhy, N.P. Transmission cost allocation by using MW-Mile approaches in a restructured Indian power system. In Proceedings of the Power and Energy Society General Meeting, Minneapolis, MN, USA, 25-29 July 2010; pp. 1-8.

6. Orfanos, G.A.; Georgilakis, P.S.; Hatziargyriou, N.D. A more fair power flow based transmission cost allocation scheme considering maximum line loading for N-1 security. IEEE Trans. Power Syst. 2013, 28, 3344-3352.

7. Shirmohammadi, D.; Gribik, P.R.; Law, E.T.K.; Malinowski, J.H.; O’Donnell, R.E. Evaluation of transmission network capacity use for wheeling transactions. IEEE Trans. Power Syst. 1989, 4, 1405-1413.

8. Gu, C.; Li, F. Long-run marginal cost pricing based on analytical method for revenue reconciliation. IEEE Trans. Power Syst. 2011, 26, 103-110.

9. Rao, M.S.S.; Soman, S.A. Marginal pricing of transmission services using min-max fairness policy. IEEE Trans. Power Syst. 2015, 30, 573-584.

10. Rubio-Oderiz, F.J.; Perez-Arriaga, I.J. Marginal pricing of transmission services: A comparative analysis of network cost allocation methods. IEEE Trans. Power Syst. 2000, 15, 448-454.

11. Nojeng, S.; Hassan, M.Y.; Said, D.M.; Abdullah, M.P.; Hussin, F. Improving the MW-Mile method using the power factor-based approach for pricing the transmission services. IEEE Trans. Power Syst. 2014, 29, 2042-2048.

12. Monsef, H.; Jaefari, M. Transmission cost allocation based on use of reliability margin under contingency conditions. IET Gener. Transm. Distrib. 2009, 3, 574-585.

13. Molina, Y.P.; Saavedra, O.R.; Amaris, H. Transmission network cost allocation based on circuit theory and the Aumann-Shapley method. IEEE Trans. Power Syst. 2013, 28, 4568-4577.

14. Pandya, K.S.; Joshi, S.K. Sensitivity and particle swarm optimization-based congestion management. Electr. Power Compon. Syst. 2013, 41,465-484.

15. Hazra, J.; Sinha, A.K. Congestion management using multiobjective particle swarm optimization. IEEE Trans. Power Syst. 2007, 22, 1726-1734.

16. Pandi, V.R.; Biswas, A.; Dasgupta, S.; Panigrahi, B.K. A hybrid bacterial foraging and differential evolution algorithm for congestion management. Eur. Trans. Electr. Power 2010, 20, 862-871.

17. Panigrahi, B.K.; Pandi, R.V. Congestion management using adaptive bacterial foraging algorithm. Energy Convers. Manag. 2009, 50, 1202-1209.

18. Singh, K.; Yadav, V.K.; Padhy, N.P.; Sharma, J. Congestion management considering optimal placement of distributed generator in deregulated power system networks. Electr. Power Compon. Syst. 2014, 42, 13-22.

19. Singh, H.; Hao, S.Y.; Papalexopoulos, A. Transmission congestion management in competitive electricity markets. IEEE Trans. Power Syst. 1998, 13, 672-680.

20. Erli, G.; Takahasi, K.; Chen L.; Kurihara I. Transmission expansion cost allocation based on cooperative game theory for congestion relief. Electr. Power Energy Syst. 2005, 27, 61-67.

21. Kiani, A.; Annaswamy, A. Wholesale energy market in a smart grid: Dynamic modeling and stability. In Proceedings of the 50th IEEE Conference on Decision and Control and European Control Conference, Orlando, FL, USA, 12-15 December 2011; pp. 2202-2207.

22. Bejestani, A.K.; Annaswamy, A. A dynamic mechanism for wholesale energy market: Stability and robustness. IEEE Trans. Smart Grid 2014, 5, 2877-2888. 
23. Papavasiliou, A.; Hindi, H.; Greene, D. Market-based control mechanisms for electric power demand response. In Proceedings of the 49th IEEE Conference on Decision and Control, Atlanta, MS, USA, 15-17 December 2010; pp. 1891-1898.

24. Tadahiro, T.; Koki, K.; Yoshiro, F.; Tomohiro, T.; Shiro, Y. Automated dinear function submission-based double auction as bottom-up real-time pricing in a regional prosumers' electricity network. Energies 2015, 8, 7381-7406.

25. Luo, Q.; Lu, L. Wheeling price calculating method in the price of direct power transmission to large customers. Electr. Power Sci. Eng. 2008, 24, 38-41. (in Chinese)

26. Kang, M.-S. Generation cost assessment of an isolated power system with a fuzzy wind power generation model. IEEE Trans. Energy Convers. 2007, 22, 397-404.

27. Frihauf, P.; Krstic, M.; Basar, T. Nash equilibrium seeking in noncooperative games. IEEE Trans. Autom. Control 2012, 57, 1192-1207.

28. Wang, Y.P.; Saad, W.; Zhu, H.; Poor, H.V.; Basar, T. A game-theoretic approach to energy trading in the smart grid. IEEE Trans. Smart Grid 2014, 5, 1439-1450.

29. Dasgupta, P.; Maskin, E. The existence of equilibrium in discontinuous economic games, I: Theory. Rev. Econ. Stud. 1986, 53, 1-26.

(C) 2015 by the authors; licensee MDPI, Basel, Switzerland. This article is an open access article distributed under the terms and conditions of the Creative Commons by Attribution (CC-BY) license (http:/ / creativecommons.org/licenses/by/4.0/). 